\title{
Application of Probabilistic Methods to Assess Risk due to Resonance in the Design of J-2X Rocket Engine Turbine Blades
}

\author{
Andrew M. Brown ${ }^{1}$, Michael DeHaye ${ }^{2}$ \\ NASA/Marshall Space Flight Center, Propulsion, Huntsville, AL 35812 \\ Steven DeLessio ${ }^{3}$ \\ Jacobs, Inc., NASA/Marshall Space Flight Center, Propulsion, Huntsville, AL 35812
}

\begin{abstract}
The LOX-Hydrogen J-2X Rocket Engine, which is proposed for use as an upper-stage engine for numerous earth-to-orbit and heavy lift launch vehicle architectures, is presently in the design phase and will move shortly to the initial development test phase. Analysis of the design has revealed numerous potential resonance issues with hardware in the turbomachinery turbine-side flow-path. The analysis of the fuel pump turbine blades requires particular care because resonant failure of the blades, which are rotating in excess of 30,000 revolutions/minutes (RPM), could be catastrophic for the engine and the entire launch vehicle. This paper describes a series of probabilistic analyses performed to assess the risk of failure of the turbine blades due to resonant vibration during past and present test series. Some significant results are that the probability of failure during a single complete engine hot-fire test is low $(\sim 1 \%)$ because of the small likelihood of resonance, but that the probability increases to around $30 \%$ for a more focused turbomachinery-only test because all speeds will be ramped through and there is a greater likelihood of dwelling at more speeds. These risk calculations have been invaluable for use by program management in deciding if risk-reduction methods such as dampers are necessary immediately or if the test can be performed before the risk-reduction hardware is ready.
\end{abstract}

\section{Nomenclature}

$\begin{array}{ll}\mathrm{CDF} & =\text { Cumulative Density Function } \\ \Phi & =\text { Damage Fraction } \\ \mathrm{FAF} & =\text { Fatigue Analysis Factor } \\ \mathrm{Ftu} & =\text { Ultimate tensile strength } \\ \mathrm{HCF} & =\text { High Cycle Fatigue } \\ \mu & =\text { mean } \\ m & =\text { mistuning response ratio } \\ \mathrm{PDF} & =\text { probability Density Function } \\ \mathrm{p}_{\mathrm{f}} & =\text { probability of failure } \\ \mathrm{RPM} & =\text { Revolutions per Minute } \\ \mathrm{S}_{\mathrm{a}} & =\text { Alternating Stress } \\ \mathrm{S}_{\mathrm{alteq}} & =\text { Alternating Equivalent Stress } \\ \mathrm{S}_{\mathrm{m}} & =\text { Mean Stress } \\ \sigma & =\text { standard deviation } \\ \zeta & =\text { equivalent viscous damping }\end{array}$

\footnotetext{
${ }_{1}^{1}$ Aerospace Technologist, Propulsion Structural \& Dynamic Analysis, ER41, AIAA Senior Member.

${ }^{2}$ Aerospace Technologist, Propulsion Structural \& Dynamic Analysis, ER41

${ }^{3}$ Senior Engineer, Jacobs ESTS, ER41
} 


\section{Introduction}

$\mathrm{T}$

he $\mathrm{J}-2 \mathrm{X}$ rocket engine is a LOX-Hydrogen derivative of the J-2 engine used in the Saturn launch vehicle program. Because of the success of the original J-2, much of its design was maintained, but a new higher thrust requirement, new materials, and new analysis requirements necessitated altering most of the components in the turbine-side flow path for both the LOX turbopump and the Fuel turbopump. Dynamic analysis has indicated resonance conditions for many of these components, including the turbine blades, which are critical due to their extremely high kinetic energy. As an example, a recently developed Campbell diagram/Factor of Safety chart of the J2-S Fuel $1^{\text {st }}$ stage blade for higher-order modes is shown in Fig. 1 (the J2-S was 1970's era J-2 derivative). This diagram identifies resonance by showing the intersection of the excitation mechanism line, which equals the number of or harmonic of the upstream flow distortions multiplied by speed, with bladed-disk natural frequencies. A modalstress plot of one of the resulting problematic modes (not to scale) is shown in Fig. 2.

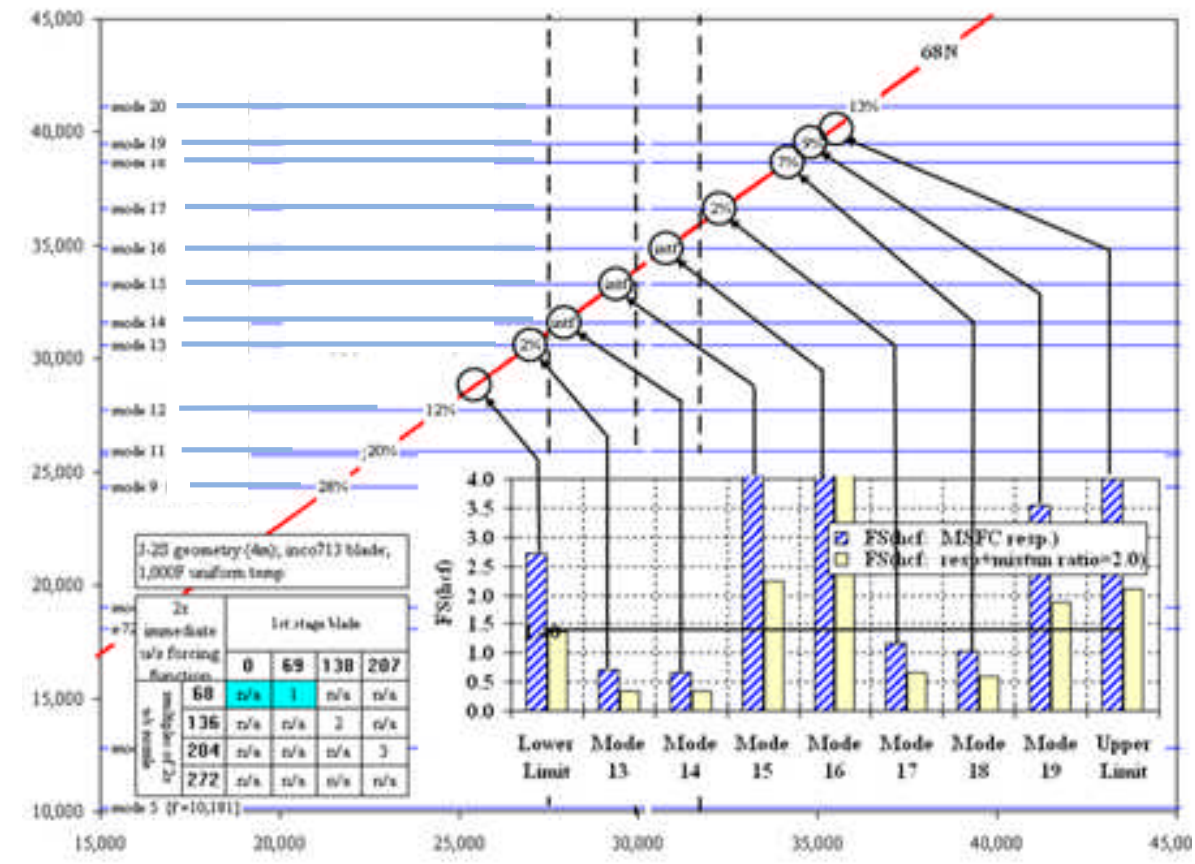

Figure 1. J-2S First Stage Campbell Diagram

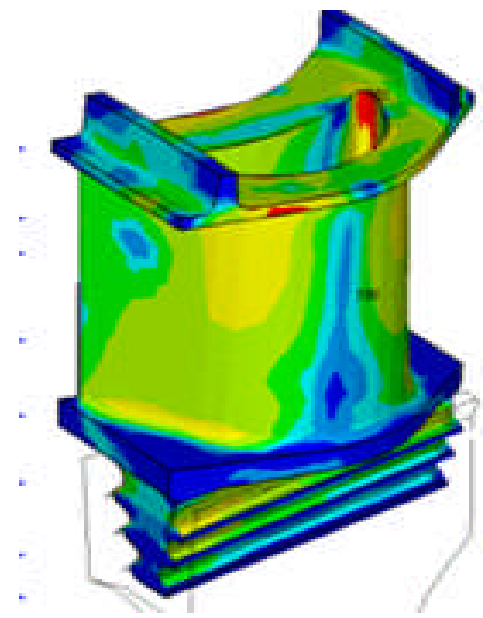

Figure 2. J-2X Turbine Blade Mode Shape (distorted geometry) 
In the past, design criteria specified that the blades and/or flow path geometry be designed such that the first few modes avoid a resonant condition. Potential resonances of higher modes were not examined. The rationale for this was that the extensive development test phase of the engine would identify any resonant issues. This philosophy has been validated in the Space Shuttle Main Engine program, where several resonant issues became evident by cracking during the development phase. These problems were dealt with during that phase by blade redesign and/or dampers, leading eventually to a successful design.

Since testing is extremely expensive, though, design criteria for new engines like the J-2X rely instead on highfidelity fluid dynamic and structural dynamic analyses of the blades to identify blade higher-order mode resonance problems and to identify methods for resolving them. This resolution requires satisfaction of the pre-determined analytical high cycle fatigue (HCF) factor of safety, which is calculated for the J-2X by a forced response analysis of the blades using computational fluid dynamics forcing functions.

The approved design criteria for this engine recognizes that many of the design parameters for blades exhibit both aleatory uncertainty, which is the inherent variation associated with the physical system, and epistemic uncertainty, which is the potential deficiency in any phase of the modeling process due to a lack of knowledge ${ }^{1}$. For instance, the forced response analysis assumes that the natural frequency could vary from the analytical prediction by $+/-5 \%$ due to an epistemic lack of knowledge of the boundary conditions and finite element inaccuracies. The J$2 \mathrm{X}$ program accounts for this conservatively by assuming an excitation within that frequency range is equal to the natural frequency (since there are extremely small levels of damping, peak response is very close to the natural frequency). Material property variations are well-recognized aleatory non-deterministic parameters. These are accounted for by using a J-2X-specific "validated" set of material properties, which is based on a certain statistical percentile of the distribution with an accepted confidence level.

During the development of the J-2X, other non-deterministic parameters became more evident than in previous programs. The first of these is amplification of peak resonant response due to mistuning, which occurs in imperfectly cyclically symmetric structures such as bladed-disks. Although this subject has been exhaustively studied in academia over the last 50 years, a practical method for prediction of the mistuning amplification $m$ in industrial turbomachinery has not existed until the last decade ${ }^{2}$. Because the geometry of the J-2X bladed-disks would likely result in a non-trivial value of $m$, a detailed study was carried out to determine this value. However, the results of any study of mistuning are non-deterministic by nature (aleatory), and only a small percentage of bladed-disks will exhibit a value of $m$ that would be problematic. Therefore, a great deal of effort was expended to try to determine and agree on an appropriate percentile from the result statistical distributions.

In addition, since the results of an early turbine blade forced response analysis showed that the acceptability of the designs was dependent on assumed levels of damping, an early "whirligig" test was performed to verify these assumptions. The whirligig test used an existing $\mathrm{J}-2 \mathrm{~S} 1^{\text {st }}$ stage fuel turbine bladed disk spun to speed by a mechanically-driven rotor and excited by a pressurized orifice plate with the number of holes arranged to excite the mode of interest. This testing showed that the assumptions were reasonable for the blade primary modes, but were high for the other modes, many of which were predicted to be resonant in the engine operating range. The whirligig results also indicated a wide-spread distribution of damping values, which made choosing an appropriate method for incorporating these statistical distributions (one for each mode) difficult.

The motivation for the study presented in this paper was driven by the need to properly assess the risk of engine operation when considering the nondeterministic nature of damping, mistuning, blade natural frequency, and engine speed. Rather than focus on deriving deterministic values for use in the design, the Mathematica $\odot$ software program was used to incorporate the effect of the entire distribution of each of these parameters into a response analysis for use in risk assessment. Analyses were run not only to predict potential failure in development testing under different scenarios (with/without dampers), but also to estimate the likelihood that the J-2S engine, which was the basis of the J-2X design, would have "failed" (actually, exhibited HCF cracking) during its demonstrated test series.

There has been substantial research and application of non-deterministic approaches to turbomachinery issues. Much of the applied effort has focused on the impact on the overall engine system by applying a "top-down" approach of combining measured reliability of similar engines. ${ }^{3}$ There has also been publicized literature focusing on a "bottoms-up" approach, i.e., assessing reliability of specific components based upon the inherent characteristics of the components. Many of these efforts focus on statistical characterization of crack growth and detailed probabilistic finite element damage analysis ${ }^{4}$, . A study by Rajagopal, et.al, uses probabilistic methods to characterize unsteady pressure loading and variable geometric and material parameters with the probabilistic finite element code NESSUS to characterize the probability density function (PDF) of the alternating stress and resulting HCF life ${ }^{6}$. 
The study presented here falls basically within the "bottoms-up" category, in that we examine the predicted probability of failure of a component based upon several critical non-deterministic parameters. Unlike general physics-based response surface techniques, which require a polynomial approximation of the solution, we were able to generate closed-form solutions as a function of our relevant non-deterministic input variables using detailed finite element forced response analysis. This is possible because the response is a simple linear function of these variables (if we assume that the random variables are independent, which is reasonable although not completely accurate). This failure equation can then be used directly in Monte Carlo and limit-state probabilistic formulations to quickly and directly generate overall probabilities of failure as a direct function of these variables. This is the first application the authors have seen to date that attempts to find the probability of failure of blades incorporating these key non-deterministic variables. The results attempt to answer the perennial questions of dynamic response analysis: "what is our chance of failure during a test series?", and for an already-built component, "why didn't we fail if the predicted resonant stresses are so high?"

\section{Discussion of Relevant Input Variables and Analysis Assumptions}

The first random variable focused on was mistuning. The J-2X is the first major rocket turbopump developed since methods have been matured enough to generate accurate estimates of $m$. Pratt $\&$ Whitney, East Hartford was contracted to perform this analysis for the J-2X first stage bladed-disk using the SNM (subset of nominal modes) $\operatorname{method}^{7}$. Statistical distributions were generated for $m$ for the three most problematic modes considering both all the blades and the maximum responding blade within a bladed-disk, yielding a total of 6 distributions. Examination of the mistuning analytical data by all the parties concerned had resulted first in a decision to adopt the traditional design percentile of a normal 3-Sigma distribution (99.865\%), but there was some disagreement over whether the individual blade or the bladed-disk statistics should be used and how to handle different statistics for different modes. The final agreement was to adopt a value of $m=2.0$ as a single approximate $3 \sigma$ value for the entire dataset (including multiple modes and blade and bladed-disk statistics). This value is consistent with the peak value reported in the literature ${ }^{8}$. The approximate average value of the mean for the 6 distributions was 0.9 , and the overall distribution was found to be lognormal, so this type of distribution with a $3 \sigma$ value equal to 2.0 was created in Mathematica $\odot$, as shown in Fig. 3. The assumption of the lognormal fit and the estimate of the parameters of that fit obviously introduce error to the calculations.
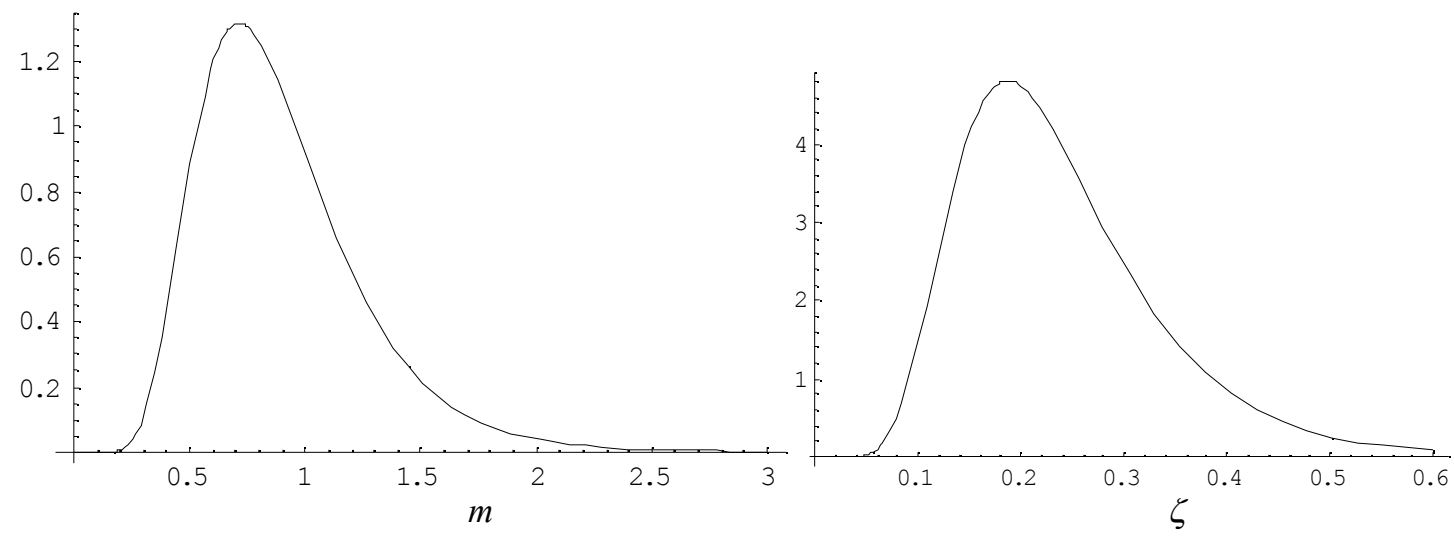

Figure 3. Distributions of Mistuning Amplification Factor $m$ and Damping Factor $\zeta$.

The other major inherently non-deterministic variable to be considered is blade damping $\zeta$ as measured in the whirligig. Unfortunately, as previously mentioned, there are some non-trivial differences between this J-2S turbine operating in the whirligig environment and a J-2X turbine operating in the actual hot-fire environment. These differences require an assumption that the damping mechanism in the whirligig will be the same as the J-2X. Additionally, the difference in the mode shapes of the different geometries requires extrapolating the damping values from the J-2S modes to the most similar J-2X modes. The extrapolation to the J-2X first stage fuel blade is the best, so the resulting error should be small in this case. Comprehensive reduction of the whirligig results produced distributions of damping for each mode, and these distributions are also best fit by a lognormal shape. A lognormal distribution for the most problematic mode's $\zeta$ with its measured mean and standard deviation was 
therefore created in Mathematica $\bigcirc$ (see Fig. 3). At this point, an important but perhaps inaccurate assumption is made that mistuning and damping are independent. As the quantification of the actual correlation of these two parameters would involve a huge effort, immediate requirements for the results of the risk analysis under pursuit by the procedure discussed here necessitated by-passing the hopefully small influence of the correlation.

A third random variable to be considered is operating speed. The gas-generator cycle of the J-2X specifies the flow rate, which when combined with the turbine efficiency will result in a rotor rotational rate. Therefore, for a generic complete engine hot-fire test with a certain engine balance and thrust level, a distribution for speed can be determined from the various engine and turbomachinery parameters. The resulting distribution is Gaussian with $\mu=30,635$ RPM and $\sigma=307.7$ RPM. However, there is also a "powerpack" type of testing in which the turbomachinery is isolated from the rest of the engine and the operating speed is moved over the entire anticipated operating range to test various conditions. A-priori prediction of the operating speed is quite difficult, and will be discussed later, but post-priori prediction consists of simply determining a desired speed bin size and tabulating the time within that bin.

The fourth random variable considered is natural frequency, where the analytical natural frequencies of the mode under consideration are taken as the means of Gaussian distributions. A rule-of-thumb combining both the aleatory and epistemic uncertainties is that $3 \sigma=+/-5 \%$ (total variation of $10 \%$ ). In order to use this distribution, the natural frequency values are converted to RPM by dividing by the appropriate engine order excitation and multiplying by 60 $\mathrm{RPM} /$ hertz. It is assumed that natural frequency is independent of mistuning, but there is probably some correlation, so this will introduce a small error.

Finally, there are a number of other parameters that possess uncertainties that will affect the analysis; the most significant ones are the fluid forcing function, the material properties, and the fluid/structure forced response analysis methodology. For simplification, the previously mentioned "validated" set of the material properties is used as a deterministic value, but is actually non-deterministic. However, for the probability assessments, the nominal curve fits of the high cycle fatigue cycles-to-failure data are used rather than the more conservative fit typically used in design. In addition, for this analysis no attempt is made to quantify the uncertainty in the forcing function or response methodology; it is believed that the deterministic values resulting from these assumptions are nominal mean type values, so their effect should be to add more error to the confidence of the probabilistic analyses rather than alter the probabilities significantly.

\section{Probabilistic Analyses}

\section{A. J-2S Powerpack I Post-Priori Probability of Failure Analysis}

A number of analyses were performed to answer risk questions posed by program management during the design phase. When it became apparent that it would be difficult to implement a damper design into the J-2X turbopump in time to make the first build of the engine for the complete-engine hot fire test, the problem to be addressed was calculating the probability of resonant failure of the most problematic turbine blade during that test. To gain confidence in this calculation, verification of the probabilistic technique was sought by using existing test data. This was achieved by calculating a post-priori probability of failure for turbine blades used in the Powerpack I test, which consisted of a J-2S turbopump-only test performed in 2009 with the objectives of proving out testing procedures.

From J-2S forced response analysis, the HCF factor for several modes was below the requirement, and the worst mode, number 14 in the $1^{\text {st }}$ nodal diameter family, had a factor of less than 1.0. The natural frequency statistical distribution is generated as described above using a $\mu=36850.7 \mathrm{~Hz}$. For damping, the most similar whirligig mode excited was number 10 of the $5^{\text {th }}$ nodal diameter family, which has a lognormal distribution with $\mu=0.304 \%$ and $\sigma=0.097 \%$.

Applying the above assumptions and distributions, a joint probability density function (PDF) in $m$ and $\zeta$ is therefore created by simply multiplying the two individual PDF's (Fig. 4). Creation of a limit state function to define failure is the next step. Both "infinite life" methods, which apply a dynamic stress based on an endurance limit, and "finite life" methods, which include stress versus cycles-to-failure curves, were considered. The final decision was to examine the finite life damage fraction, which, based upon a given service time, gives a normalized amount of damage compared to the allowable damage, so values larger than one indicate failure. Using previously determined states of stress during peak resonance in the blades from a forced response analysis, a closed form solution for damage fraction $\Phi$ as a function of $\zeta$ and $m$ is generated. At this point, we assume that the vast majority of damage occurs due to the response of this single mode. This assumption will introduce some under prediction 
error of the final probability. The damage fraction $\Phi$, which is the ratio of the accumulated cycles $\mathrm{N}_{\text {accum }}$ to the allowable cycles $\mathrm{N}_{\text {fail }}$, defines the limit state, with $\Phi>1.0$ being failure.

$$
\Phi=\frac{N_{\text {accum }}}{N_{\text {fail }}}
$$

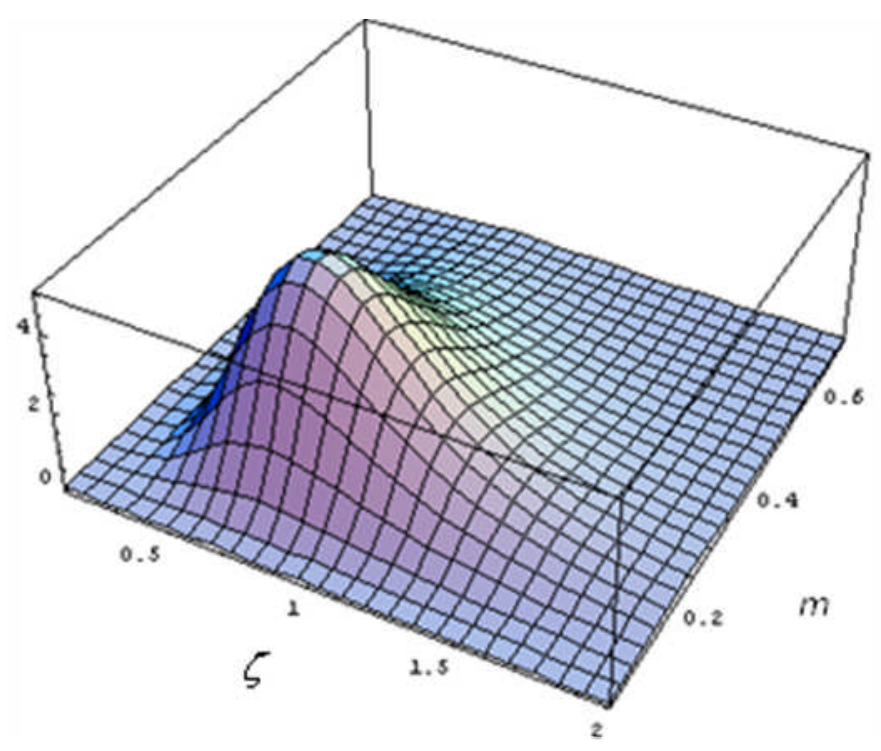

Figure 4. Joint PDF of Damping and Mistuning

The first step in generating this value is to express an equation for alternating equivalent stress $S_{\text {alteq, }}$ which is the alternating stress at an R-ratio of minus one that would result in equivalent damage to the mean and alternating state of stress that exists in the actual component. This formulation allows for direct comparison with the endurance limit. The alternating equivalent stress is therefore a function of the finite element resultant alternating stress at resonance $S_{a}$, the fatigue analysis factor FAF, which is a "fudge" factor taking into account uncertainties for design, the mean stress $\mathrm{S}_{\mathrm{m}}$, the damping $\zeta$, the mistuning factor $m$, and the ultimate tensile strength Ftu. $\mathrm{S}_{\mathrm{a}}$ is scaled for each case in the probabilistic analysis by multiplying it by the ratio of the damping value used in the original finite element analysis, 0.0025 , to the damping for that case. FAF is set equal to the nominal value of 1.0 for this analysis, since the goal is to predict a post-priori risk of failure, i.e., what is the best estimate of what actually happened, as opposed to a conservative estimate that would typically be used for design.

$$
A_{e q}=\frac{(F A F) m\left(\frac{0.0025}{\xi}\right) S_{a}}{1-\frac{S_{m}}{F t u}}
$$

Since we have assumed that the damage is essentially due to a single mode, a single-degree of freedom representation of the dynamic magnification factor is used to take into account off-resonance conditions. Equation (2) is altered to give us an expression for $A_{\text {eq }}$ at every instantaneous speed by simply scaling it by the ratio of the dynamic magnification factor to the peak value of the magnification factor: 


$$
A_{e q}=\frac{S_{a} * F A F * m * \frac{.0025}{0.01 \zeta} * \frac{\sqrt{\frac{1}{\left(\left(1-\left(\frac{\text { speedrpm }}{\text { fnrpm }}\right)^{2}\right)^{2}+\left(\frac{2(0.01) \zeta * \text { speedrpm }}{\text { fnrpm }}\right)^{2}\right.}}}{1-\frac{1}{2(0.01) \zeta}}}{1-\frac{S_{m}}{F_{t u}}}
$$

$\mathrm{A}_{\text {eq }}$ is then plugged into the nominal curve fit of HCF cycle count data to obtain $\mathrm{N}_{\text {fail }}$.

$$
N_{\text {fail }}=10^{\left(-9.2461 \times \log _{10}\left(A_{e q}\right)+20.672\right)}
$$

$\mathrm{N}_{\text {accum }}$ is equal to the excitation frequency multiplied by time at that frequency, so we will need to calculate the time spent at each location on the magnification curve defined above. The speeds for the test series were recorded and tabulated into bins of 80 RPM. An incremental damage fraction covering the damage occurring within the bin as a result is desired. It must be assumed that the speed is uniformly distributed within each bin (lacking data which would give more detail); this will yield the same result as stating that the speed will ramp linearly through the bin over the measured time within the bin. (As an aside, even though much of the recorded time within the bins was actually at a single speed "dwell", rather than a ramp, the speed actually will be both drifting slightly and will be "dithering" (oscillating) about that speed", so the continuous ramp assumption is reasonable.) Therefore, we first obtain the instantaneous speed vector

where

$$
\{\text { speedramps }(h z)\}=\{\text { binstarts }(h z)\}+\{\text { ramprates }(h z / s)\}
$$

$$
\{\text { binstarts }(h z)\}=\{\text { speed at beginning of bin }(\text { rpm })\} *
$$

and

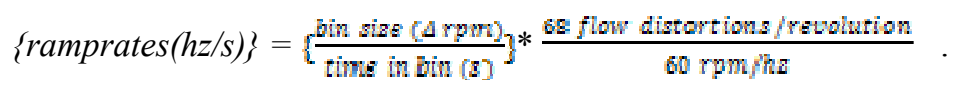

We now express the incremental damage fraction as the ratio of the speed to $\mathrm{N}_{\text {fail }}$ and integrate with respect to time to get the total damage for the i'th bin:

$$
\Phi_{i}=\int_{0}^{\text {time }_{i}} d \Phi=\int_{0}^{\text {time }_{i}} \frac{\text { speedramps }_{i}}{N_{\text {fail }}} d t
$$

The quantity to be solved for is the probability that the damage fraction is below some value for each speed bin. This can be obtained by integrating the volume underneath the joint PDF up to the limit state or performing Monte Carlo analysis (see Fig. 5). The limit state is obtained by solving for one of the random variables in terms of the others. In this case, solving for $m$ yields an extremely complicated polynomial in terms of each of the other random variables. The exact integration was performed successfully for two-dimensional cases examined earlier in the investigation when only $m$ and $\zeta$ were considered, but that integration was intractable when considering all four random variables, so Monte Carlo is performed instead. In order to obtain the damage fraction for each sample of the random variables $m, \zeta$, natural frequency, and speed, the damage fraction for each speed bin is calculated individually as shown in equation (6) and the fractions summed for the entire speed range for that sample.

$$
\Phi_{\text {total }}=\sum_{i=1}^{\text {number of bins }} \Phi_{i}
$$

This calculation was quite lengthy and could not be entirely automated, but a reasonable sample size of 500 samples was able to be executed, resulting in a $1 \%$ failure rate. This value identified the fact that although the 
predicted HCF factor was below 1.0, the actual chance that the blade would have failed during the PowerPack I test series was low.

\section{B. J-2S Powerpack I A-priori Analysis}

During the review of the J-2S Powerpack I Post-priori analysis, a question was raised as to the validity of the prediction technique for application in an a-priori sense, where the measured speed bin data would not be available. To answer this question, an a-priori analysis effort was initiated by making assumptions concerning how the test would be performed. Discussions with turbomachinery engineering and management resulted in the following assumptions: 1200 seconds of total run time, consisting of 4 dwells of 100 seconds, 20 dwells of 30 seconds, and ramping from $26902 \mathrm{RPM}$ to $31200 \mathrm{RPM}$ at a continuous rate of $20 \mathrm{RPM} / \mathrm{sec}$.

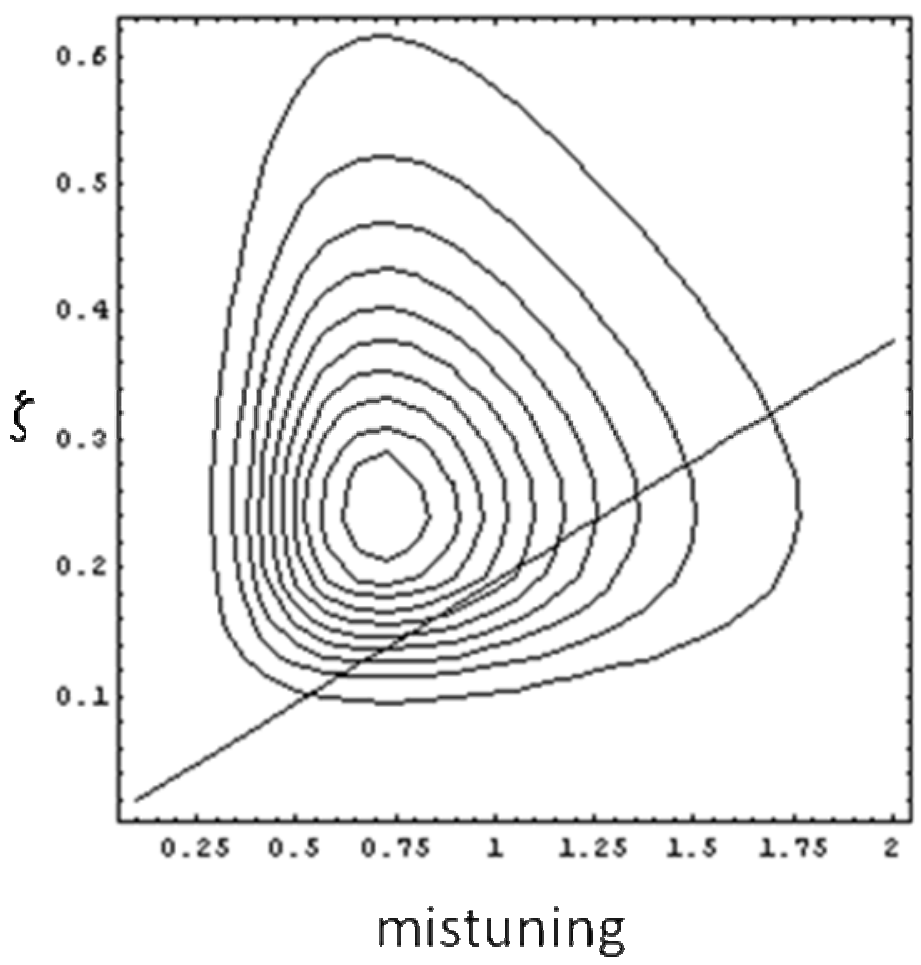

Figure 5. $\mathrm{P}_{\mathrm{f}}$ is integration of volume up to limit state

The initial part of this analysis is identical to the post-priori analysis. For the last step, though, the process is different. Since each Monte Carlo sample specifies a specific time of service at a specific speed, the damage fraction for each of the given dwell times is simply calculated using equation (1), with

$$
\left\{N_{\text {accum }}\right\}=\{\text { speed }(R P M)\} * \frac{6 \text { flow distortions/rowiution }}{60 \text { rom/haz }} *\{\text { well time }(s)\} \text {. }
$$

500,000 sample simulations yielded a probability of failure of 0.00124 for each of the four 100 second dwells and 0.000662 for the 30 second dwells. The probability for the assumed ramps is calculated exactly as it was done for the post-priori test, only the rate of $20 \mathrm{RPM} / \mathrm{sec}$ is used instead of $80 \mathrm{RPM} /$ measured bin time, and the integral is performed over the entire speed range of the test instead of broken up into bins. The resulting probability for the ramps is 0.016 . Since the probability of failure either during the dwells or the ramps are independent events, the law of total probability states that the component probabilities should be summed, thereby yielding a total probability of $3.42 \%$. Although higher than the actual probability of failure for the test using the post-priori measured speed bins, this result provided credibility that the technique could be used ahead of time for prediction and that the results would be at the same order of magnitude. 


\section{J-2X Powerpack II A-priori Analysis}

One of the main purposes of the probabilistic technique could now be applied, which was to predict a probability of blade failure for the J-2X powerpack II series, which will be performed using the J-2X turbine after it is built. The question in particular is how critical it is for blade dampers to be implemented into the turbine. This analysis follows the Powerpack I a-priori procedure. For this test, the critical mode is different, so the damping distribution is as well, and the dwell and ramp times assumptions are slightly different. The resulting probability of failure, $\mathrm{p}_{\mathrm{f}}$, for the J-2X Powerpack II is $33.8 \%$, which is much higher than the original J-2S Powerpack I test. This is because the $\mathrm{J}-2 \mathrm{~S}$ problematic mode has three times as much damping as the $\mathrm{J}-2 \mathrm{X}$ mode, and because the operating range and operating time of Powerpack I was much less than Powerpack II will be. In addition, the Powerpack II operating range completely encompasses the natural frequency range of the problematic mode, so a resonant condition will definitely be reached at least during the ramping portions of the test (see Fig. 6).

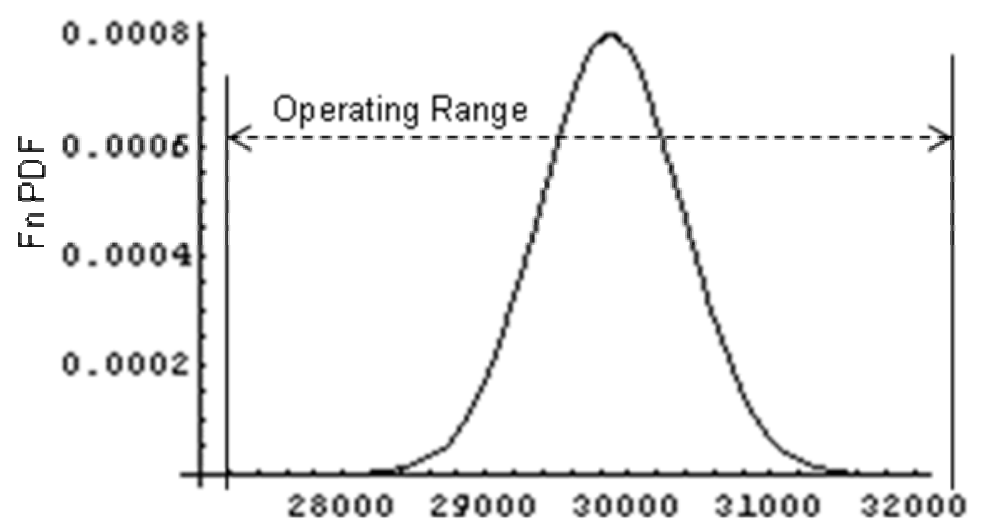

Figure 6. PDF of Natural frequency in RPM of J-2X $1^{\text {st }}$ St. Fuel Blade Problematic mode overlapped by Powerpack II Operating Range

\section{J-2X Engine I A-priori Analysis}

An equally important analysis was to assess the blade $p_{f}$ for the first full-scale engine test, which will be performed at around the same time as the Powerpack II test and so will also have difficulty implementing the dampers. For this case, we evaluate the $\mathrm{p}_{\mathrm{f}}$ for engine operation resolving to a single speed during an entire test of $550 \mathrm{sec}$. The PDF of the speed is expected to follow a Gaussian distribution with a $\mu=30635$ RPM and a $\sigma=307$ RPM. Using $550 \mathrm{sec}$ as the input timeservice variable, a 100,000 sample Monte Carlo solution for the damage fraction is performed, yielding a $\mathrm{p}_{\mathrm{f}}=1.06 \%$. To understand the result, the $\mathrm{p}_{\mathrm{f}}$ values associated with failure at resonance (where resonance is defined with a half-power range and the speed is dithering, thereby increasing the life by a factor of 2.5, as reported in previous reference by Davis, et. al.) and the probability of resonance itself were separately calculated. This showed a $\mathrm{p}_{\mathrm{f}}$ given the dithering resonance of $63 \%$, but a probability of resonance of only $3.1 \%$, so the total probability product is $2 \%$. This final result is different than the first one because of the dithering assumption, but illustrates that the main reason resonant failure probability is low is because the probability of resonance itself is low.

\section{Conclusions}

A framework procedure has been established for calculating probabilities of failure for turbine blades in a variety of configurations and test programs. The random variables considered in the analysis were mistuning, damping, natural frequency, and speed. Several assumptions were necessary to enable the analyses to be tractable, including that mistuning and damping are natural frequency independent. The results have been extremely useful to project management in making component-level risk assessments for a specific engine configuration or test series.

This analysis is the first seen in the literature taking the approach of using a single point-level dynamic finite analysis result that is a function of random variables to produce probabilistic results. It is also the first to use the 
random variable set described above. The framework identified has many other applications in this field, as well. For instance, a procedure using this framework has been used to find a single deterministic value of damping that, when combined with the mistuning distribution, represents the combined three sigma probability level of the data. This procedure will be discussed in a future paper.

\section{References}

\footnotetext{
${ }^{1}$ Oberkampf, W. L., "Introduction to Verification and Validation," Verification and Validation of Computational Fluid Dynamics, von Karman Institute for Fluid Dynamics, Rhode, Saint Genese, Belgium, 2000

${ }^{2}$ Castanier, M. P., Pierre, C., "Modeling and Analysis of Mistuned Bladed Disk Vibration: Status and Emerging Directions, Journal of Propulsion and Power, Vol. 22, No. 2, March -April 2006

${ }^{3}$ Packard, M. H., "The Use of Probabilistic Methods to Evaluate the Systems Impact of Component Design Improvement on Large Turbofan Engines," NASA/CP_-2002-211682, 2002

${ }^{4}$ Brown, A. M., "Development of a Probabilistic Dynamic Synthesis Method for the Analysis of Non-Deterministic Structures, Ph.D. Dissertation, Georgia Tech, 1998

${ }^{5}$ Petrov, E. P. , "A Sensitivity-Based Method for Direct Stochastic Analysis of Nonlinear Forced Response for Bladed Disks With Friction Interfaces", Journal of Engineering for Gas Turbines and Power, Vol. 130, March 2008

${ }^{6}$ Rajagopal, K.R., DebChaudhury, A., Orient, G., "Demonstration of the Application of Composite Load Spectra (CLS) and Probabilistic Structural Analysis (PSAM) Codes to SSME Heat Exchanger Turnaround Vane”, NASA/CR-2000-210236, June 2000

${ }^{7}$ Yang, M.T., Griffin, J.H.,’A Reduced-Order Model of Mistuning Using a Subset of Nominal System Modes,” Journal of Engineering for Gas Turbines and Power, October 2001, Vol. 123, p. 893-900

${ }^{8}$ Koul, A. K., et.al., "Improving Component Life Prediction Accuracy and Reliability Through Physics Based Prognosis - A Probabilistic Turbine Blade Case Study," Proceedings of the ASME Turbo Expo 2008, June 9-13, Berlin, Germany

${ }^{9}$ Davis, R. B, Durham, R.C., Brown, A. M., "The Response of a Mechanical Oscillator Due to Swept and Dithered Excitation”, 51st AIAA Structures, Structural Dynamics, and Materials Conference, Orlando, Fl., April 12-15, 2010
} 
Application of Probabilistic Methods to Assess Risk due to Resonance in the Design of J-2X Rocket Engine Turbine Blades

Andrew M. Brown, Michael DeHaye, Steven DeLessio

NASA/Marshall Space Flight Center/Propulsion Structural \& Dynamic Analysis

AIAA $52^{\text {nd }}$ SDM Conference

April 5, 2011 


\section{Agenda}

- Introduction \& Motivation

- Input Variables \& Assumptions

- Probability of Failure Analyses

- J-2S Powerpack I Post-Priori

- Incorporation of Finite Life Calculation

- J-2S Powerpack I A-Priori

- J-2X Powerpack II A-priori

- J-2X Engine I A-Priori Analysis

- Conclusions and Follow-On Work 
- NASA \& Pratt Whitney Rocketdyne designing J-2X Rocket Engine to be upper stage engine of some future launch system.

- $\mathrm{J}-2 \mathrm{X}$ is derivative of Saturn J-2 and follow-on J-2S, but new requirements required alteration of much of turbomachinery components and flows.

- New analysis-based design and verification introduced consideration of higherorder modes, which showed resonance.

- Resonance requires forced response analysis, which resulted in low HCF FoS.

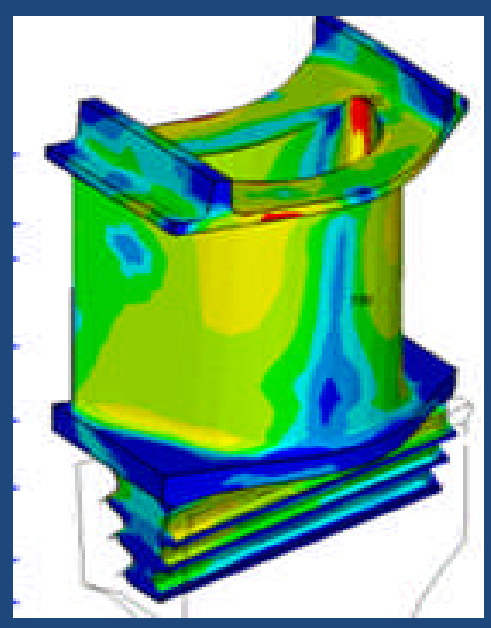

J-2X Turbine Blade Mode Shape (distorted geometry)

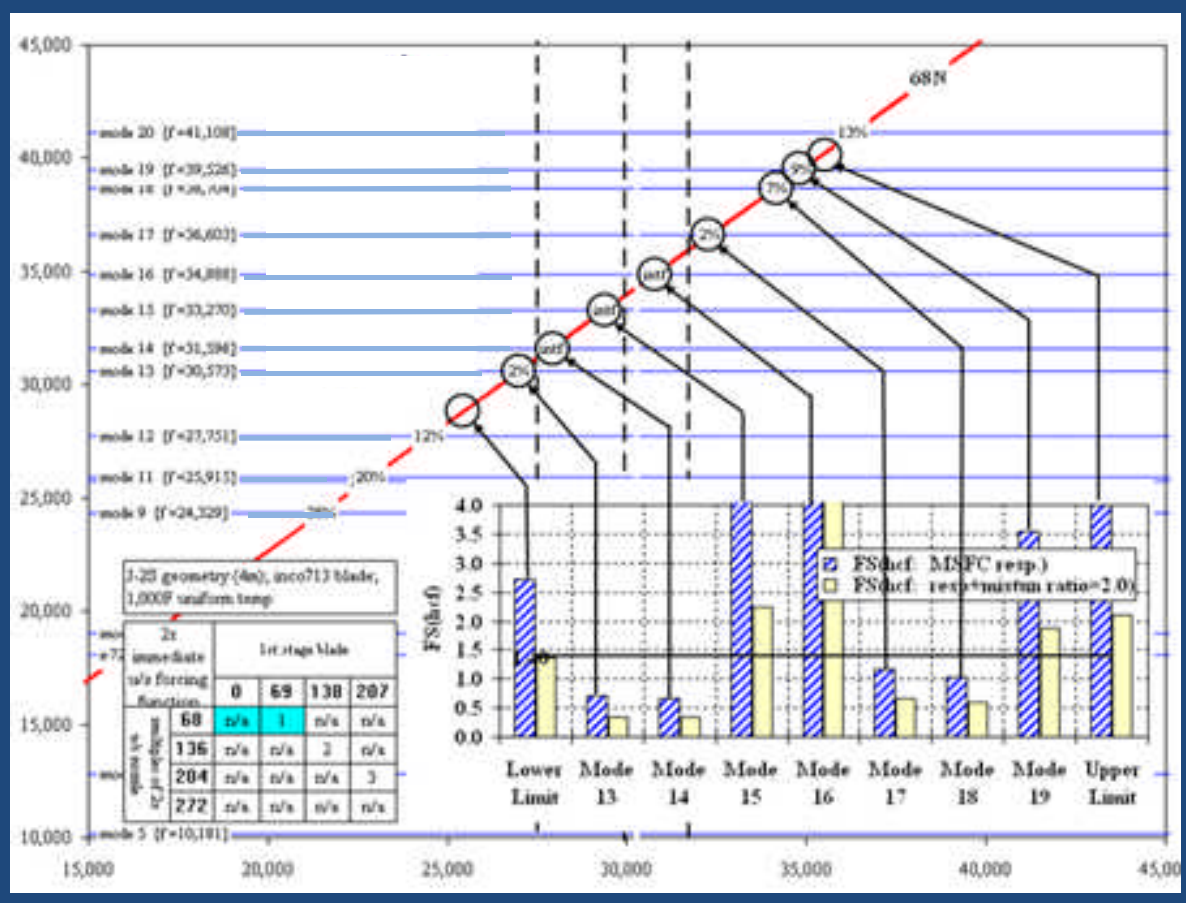

J-2S First Stage Campbell Diagram 


\section{Motivation}

- Standard blade forced-response analysis process recognizes aleatory uncertainty in material properties and epistemic uncertainty in prediction of natural frequencies.

- During J-2X design, became clear that other non-deterministic variables, damping and mistuning, would also play major role.

- Need arose to properly assess risk of blade failure using actual non-deterministic nature of these random variables rather than using deterministic value of each used for design.

- Substantial research and application in literature of probabilistic methods to turbomachinery issues

- Much of effort ("top down") calculates reliability by comparison to measured reliability of sub-systems on similar engines - Packard, ' 02.

- Statistical characterization of crack growth in probabilistic FEA ("bottoms-up) - Petrov, '08.

- Rajagopal, '00, characterized unsteady pressure loading, geometry variation, mat'l properties in NESSUS to get dynamic stress ("bottoms-up").

- Here, we calculate probability of failure using closed-form finite life solutions in terms of these 4 non-deterministic variables and peak FEA-derived stress state. 


\section{Input Variables \& Assumptions}

- Variation of Natural Frequency fn of problematic modes is typically accounted for by using rule-of-thumb of $+/-10 \%$ in frequency response analysis.

- Here, we examined data from previous engine programs and saw distribution is somewhat Gaussian with a $3 \sigma$ variation of $+/-5 \%$ ( $\rho=1.67 \%)$.

- For application, fn converted to speed by dividing by excitation order of engine speed and multiplied by $60 \mathrm{rpm} / \mathrm{hz}$.

- Operating Speed also can be considered random variable, especially for gas-generator rocket engine cycle where flow rate, turbine efficiency determine rotor rotational rate.

- For the engine balance used here, resulting operating speed distribution is

$$
\text { Speed } \sim N(\mu=30,635 \mathrm{rpm}, \sigma=307.7 \mathrm{rpm})
$$

- Exception is in "Powerpack" testing, where turbopumps are isolated and rate is controlled. 


\section{Mistuning}

- Imperfectly cyclically-symmetric structures like real bladed-disks exhibit warping of nodal-diameter modes and amplification (mistuning) of the peak response compared to a mathematically perfect cyclic-symmetric (tuned) disk.

- Effects of mistuning non-deterministic since every build of a bladed-disk will be different.

- $\mathrm{J}-2 \mathrm{X}$ is one of first rocket turbopumps developed since a practical methods developed to predict the statistics of the mistuning amplification value $m$.

- Analysis performed by M. T. Yang, P\&W E. Hartford, using his "SNM" method, to develop statistics of $m$ for 3 of J-2X problematic modes.

- Stats developed for entire-blade population $(690,000)$ and max-responding blade in blade-disk population $(10,000),->6$ distributions.

- Debate, agreement to adopt a value of $m=2.0$ as a single approximate $3 \sigma$ value for the entire dataset.

- For probabilistic analysis, approximate mean value is 0.9 with Lognormal fit assumed.

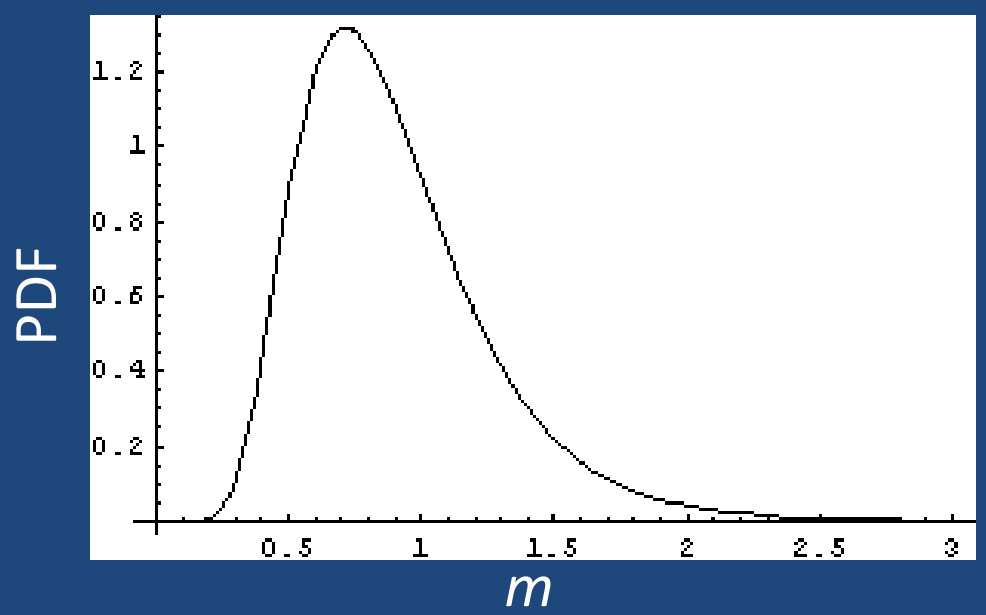




\section{Damping}

- Damping is critical parameter for forced response prediction, so "whirligig" test program used to obtain data.

- Whirligig is mechanically-driven rotor with bladed-disk excited by pressurized orifice plate simulate blade excitation. Key assumption is that this reflects true configuration.

- Data shows wide-variation in damping, but reasonable population (30-50 data values) for statistical characterization.

- Lognormal distribution fits obtained for each mode.

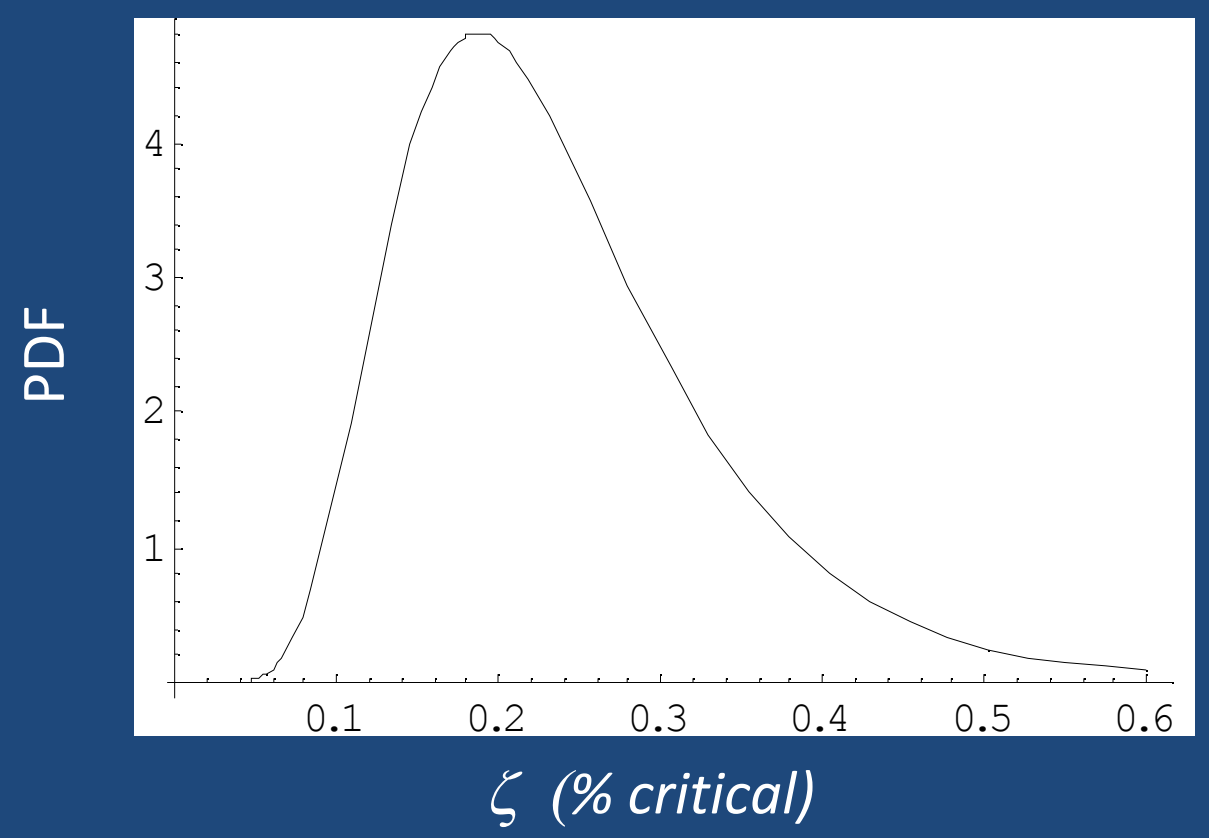




\section{J2-S Powerpack I Post-Priori $P_{f}$ Analysis}

- Became apparent that implementation of external damper needed to show design good for deterministic analysis would be behind schedule.

- Goal was to use probabilistic analysis to show $P_{f}$ during individual tests in series.

- First had to verify technique by examining probability that blades should have failed during test of J-2S system, which had FoS below 1.0, but didn't fail.

- Problematic mode 14 in ND 5 family:

$$
\text { - fn N(36851 hz, } 615 \mathrm{hz}), \zeta \sim \operatorname{LN}(0.304 \%, 097 \%) \text {. }
$$

- Failure defined using finite life approach; seek $N_{\text {accum }}$, accumulated cycles at stress state, and $\mathrm{N}_{\text {fail }}$, number of cycles at that stress state that will cause failure:

$$
\Phi=\frac{N_{\text {accum }}}{N_{\text {fail }}}>1
$$




\section{Finite Life Calculation}

- Stress state (Alternating stress Sa, Mean stress Sm) of problem location determined from frequency response finite element analysis at resonance.

- $1^{\text {st }}$ step, calculate Equivalent Alternating Stress $A_{\text {eq }}$ :

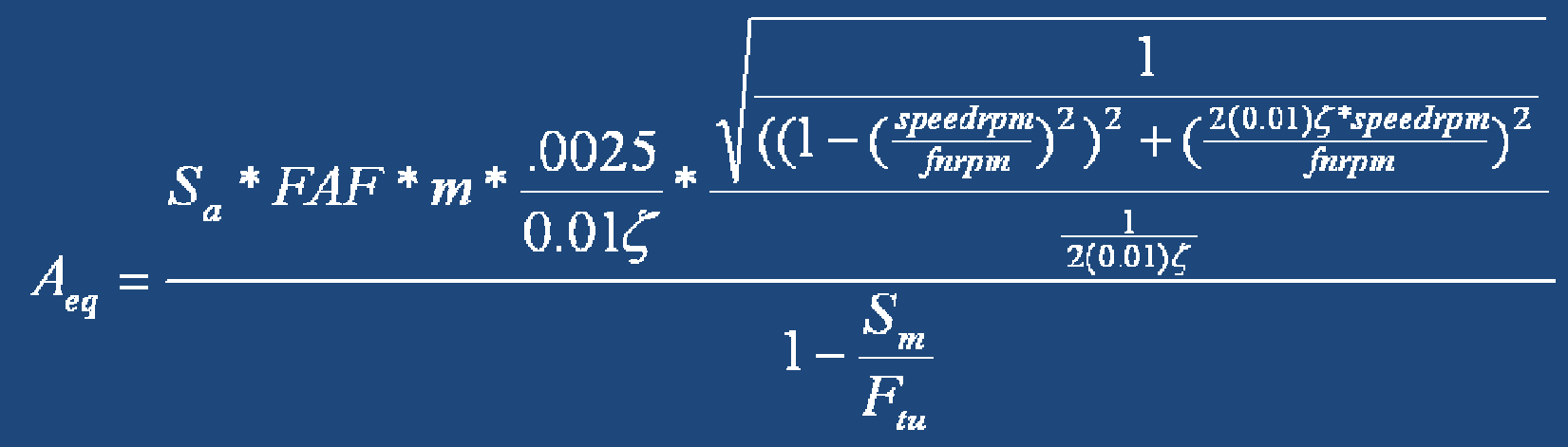

- HCF cycle count data ->

$$
N_{\text {fail }}=10^{\left(-9.2461 \times \log _{10}\left(A_{e q}\right)+20.672\right)}
$$


- $\mathrm{N}_{\text {accum }}$ is excitation frequency $*$ time at that frequency. Speeds in test series recorded in $80 \mathrm{rpm}$ wide bins, desire incremental damage fraction within each bin. Have to assume speed uniformly distributed within each bin (no other info available), same as speed ramping linearly through bin.

$\{$ speedramps $(h z)\}=\{$ binstarts $(h z)\}+\{$ ramprates $(h z / s)\}$

$\{$ binstarts $(\mathrm{hz})\}=\{$ speed at beginning of bin $(\mathrm{rpm})\} * \frac{68 \text { flow distortion s/rev }}{60 \mathrm{rpm} / \mathrm{hz}}$ $\{$ ramprates $(\mathrm{hz} / \mathrm{s})\}=\frac{\text { binsize }(\text { drmp })}{\text { time in } \operatorname{bin}(\mathrm{s})} * \frac{68 \text { flow distortions } / \text { rev }}{60 \mathrm{rpm} / \mathrm{hz}}$

- Incremental damage fraction is integral of (speedramps) dt for each bin

$$
\Phi_{i}=\int_{b}^{\text {ime }} d \Phi=\int_{0}^{i m e_{i}} \frac{\text { speedramps }_{i}}{N_{\text {fail }}} d t
$$




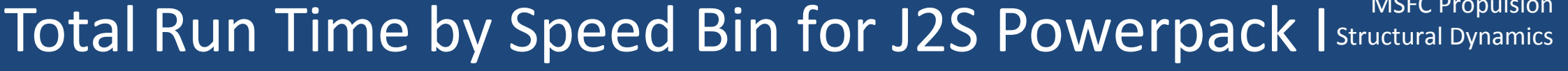

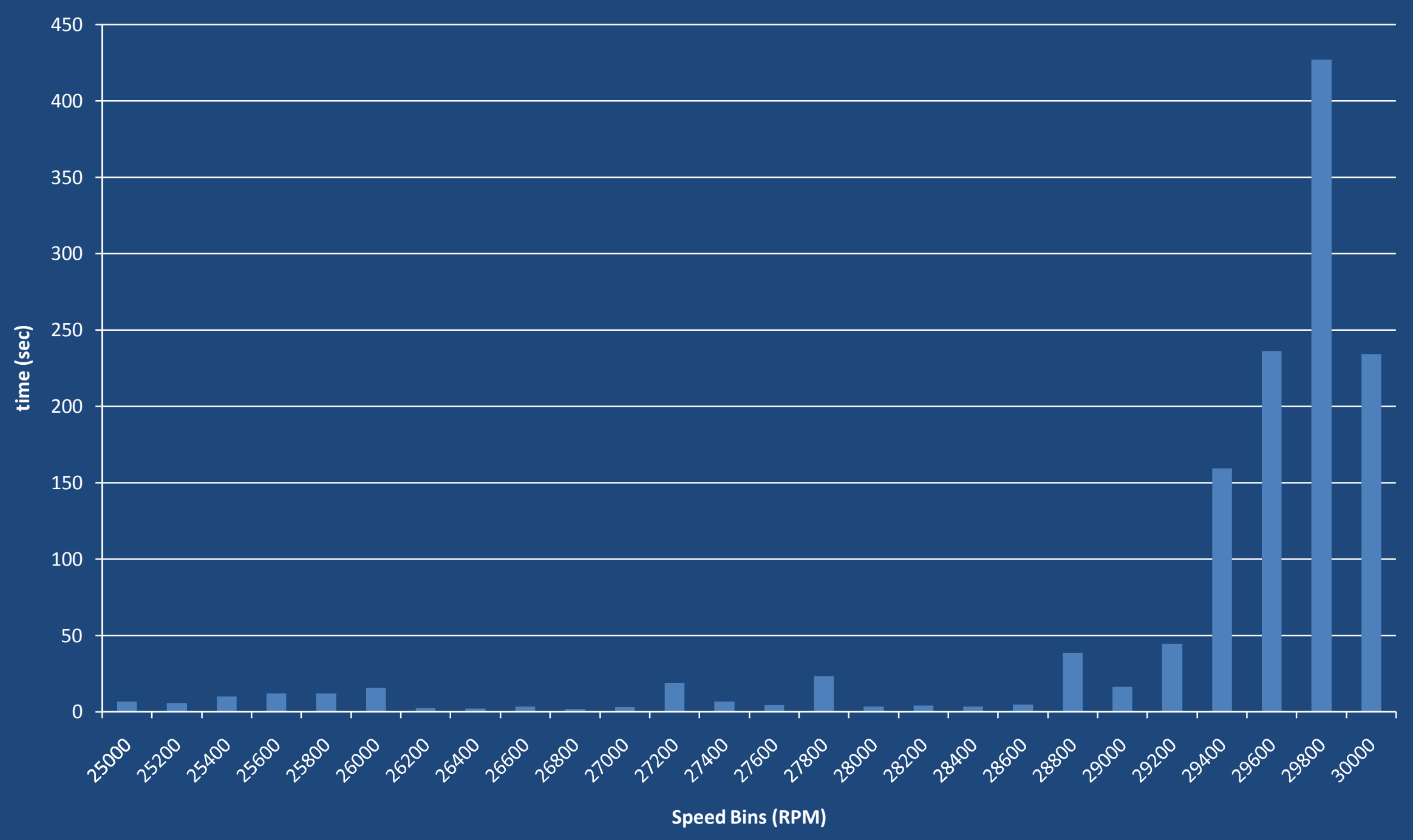




\section{Monte Carlo Analysis}

- Total Damage:

$$
\Phi_{\text {total }}=\sum_{i=1}^{\text {number of bins }} \Phi_{i}
$$

- $P_{f}$ could be determined by integrating failure state as defined up to limit state, or Monte Carlo.

- Integration achieved only considering $m$ and $z$, but intractable for all 4 r.v.'s.

- Therefore, samples of each r.v. obtained from assumed distribution, inserted into damage fraction integration and summation, and total damage fraction compared to 1 .

- $P_{f}$ is just ratio of those occurances that exceed 1 to total number of samples.

- Integration req'd for each bin for each sample, limiting MC analysis to 500 samples.

- $P_{f}=1 \%$.

- Conclusion is that although predicted deterministic HCF FoS $<1$, very low chance blade would have actually failed during the given test. This is mainly due to low probability of resonance. 


\section{J-2S Powerpack I A-priori Analysis}

- Question during review of previous analysis as to validity in a predictive sense (speed bin data would not be available).

- Engineering team made following assumptions: $1200 \mathrm{~s}$ total run time, 4 dwells of $100 \mathrm{~s}, 20$ dwells of $30 \mathrm{~s}$, ramping from $26902 \mathrm{rpm}-31200 \mathrm{rpm}$ at continuous rate of $20 \mathrm{rpm} / \mathrm{s}$ (during ramps).

- Mostly same as previous analysis, except $N_{\text {accum }}$ calculation done differently for dwells only (calculation for ramps same, except instead of over different bins just over entire range at $20 \mathrm{rpm} / \mathrm{sec}$ instead of $80 \mathrm{rpm} / \mathrm{meas}$. bin time); for dwells:

$$
\left\{N_{\text {accum }}\right\}=\{\text { speed }(R P M)\} * \frac{68 \text { flow distortions } / \mathrm{rev}}{60 \mathrm{RPM} / \mathrm{h} z} *\{\text { dwell time }(\mathrm{s})\}
$$

- Simpler calculation enabled 500,000 sample MC run:

\begin{tabular}{|l|l|}
\hline Type of operation & Pf \\
\hline 30 sec dwell & .000662 \\
\hline $100 \mathrm{sec}$ dwell & .00124 \\
\hline Ramp over entire range & .016 \\
\hline
\end{tabular}

- Total $P_{f}=\Sigma P_{f}$ independent events, so total $P_{f}=3.42 \%$, still low, technique valid. 
- Goal of probabilistic technique now achievable, to evaluate risk in test series to be performed last 2011.

- Pf was used to assist with decision to implement external blade dampers in test.

- Procedure follows J-2S Powerpack I A-Priori procedure.

- Different critical mode (-> different damping), different dwell \& ramp time assumptions.

- Resulting $P_{f}=33.8 \%$

- Explanation for results is extensive overlap of fn distribution over operating range, ensuring resonance (unlike previous Powerpack I)

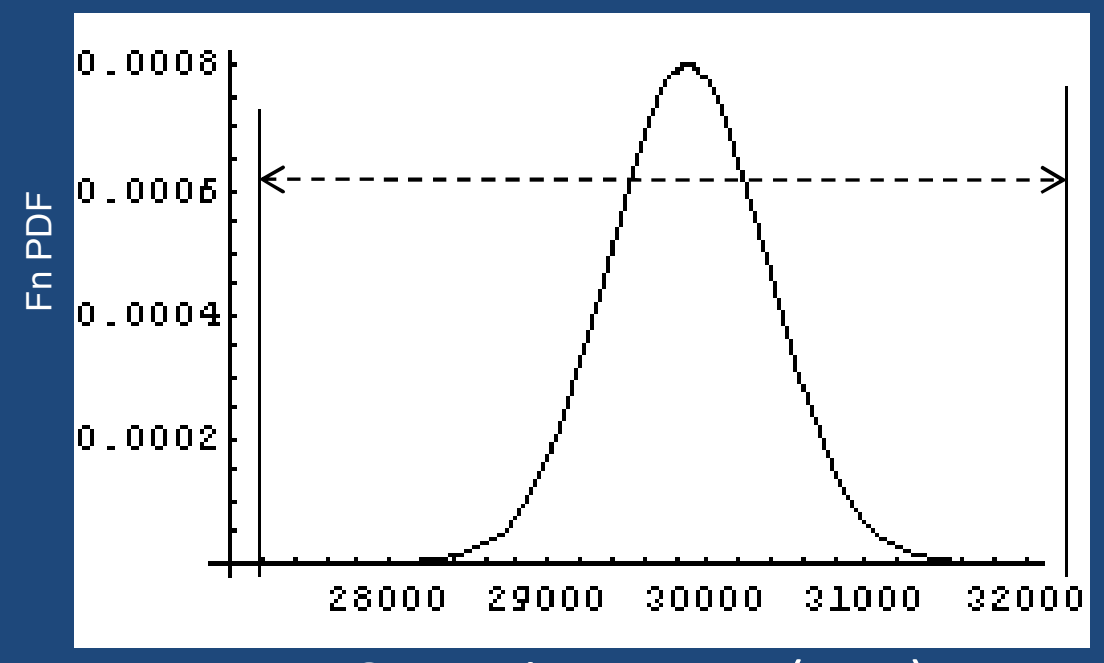

Operating Range (rpm) 


\section{J-2X Engine 10001 A-Priori Analysis}

- Equally important to assess $P_{f}$ for first full-scale engine test to determine if external blade dampers required.

- In this test speed will resolve to a single value within distribution following Speed N(30635 rpm, 307 rpm).

- Time of operation given as $550 \mathrm{~s}$.

- Single dwell formulation relatively simple, enables 100,000 sample MC run.

- $P_{f}=1.06 \%$, very low because of low probability of resonance itself, which was independently calculated to be $P_{\text {resonance }}=3.1 \%$. 


\section{Conclusions and Future Work}

- Framework procedure established for quantifying risk of turbine blade failure due to resonance.

- Probabilistic analysis enable first-time use of statistical distributions of most of random variables, including Natural Frequency, Operational Speed, Mistuning, and Damping.

- Other input variables assumed to be deterministic:

- Loading, assumed to be nominal so should not effect results significantly.

- Material properties, deterministic value are conservative, so should cause slight overprediction of Probability of Failure predictions.

- Mistuning and Damping assumed to be independent and they probably are not; unknown effect on results.

- Results very useful for project decision-making during design phase of program.

- Framework has also been applied to find a single deterministic value of damping that, when combined with the mistuning distribution, represents the combined three sigma probability level of the data (future paper). 